\title{
Commentary on Comparing Timeline Rhythms in Pygmy and Bushmen Music by Adrian Poole
}

\author{
VICTOR GRAUER [1] \\ Independent Scholar
}

\begin{abstract}
In his fascinating paper, "Comparing Timeline Rhythms in Pygmy and Bushmen Music," Adrian Poole offers a new perspective on an old debate regarding the historical significance of the many apparent affinities between African Bushmen and Pygmy music by focusing on "the analysis of one central musical feature that has received little attention in the literature: timeline rhythms." What makes this topic particularly intriguing is the way in which such rhythmic patterns lend themselves to mathematical analysis, a property which enables Poole to produce a very interesting phylogenetic tree from a simple algorithm. His thorough comparative study of these very distinctive rhythms, as found among African hunters isolated for thousands of years in remote regions of that continent with little likelihood of mutual influence, represents a significant contribution to our understanding of certain basic principles underlying both African and African-American rhythms, with additional insights relating to the "deep history" of the timeline on that continent and beyond.
\end{abstract}

Submitted 2016 December 19; accepted 2017 July 30.

KEYWORDS: timeline, rhythm, Pygmy, Bushmen, African music

AS Adrian Poole reminds us, ethnomusicologist Gilbert Rouget first called attention to "striking resemblances between Pygmy and Bushmen music" back in the 1950's, "and speculated that they share a common cultural heritage." Since then, there has been "considerable debate amongst music scholars," myself included, regarding the historical significance, if any, of the many apparent affinities between Bushmen and Pygmy music generally, based on the comparative study of a wide array of different stylistic features. In this paper, Mr. Poole offers "a new perspective on this debate" by narrowing his focus to "the analysis of one central musical feature that has received little attention in the literature . . : timeline rhythms."

Rhythmic patterns typically beaten out on a certain type of iron bell or similar instrument are commonly used to keep time in many genres of African and African-American music. Since the smelting of iron is thought to have originated independently among Bantu-speaking people in West Africa, the tradition may have spread from there to the rest of the continent via the so-called "Bantu expansion," thought to have begun roughly 3,000 years ago. Although various Pygmy and Bushmen groups, now based largely in central and southwest Africa respectively, lacked metal tools or instruments until recently, rhythmic patterns strongly resembling the Bantu bell patterns, produced by handclaps or the beating of rattles or sticks, are an essential part of their musical traditions as well. Since the two hunter-gatherer groups are thought to have been effectively isolated from one another for many thousand years, the possibility arises that these patterns may go very far back into the depths of African history, well before either the invention of iron or the origins of the Bantu languages. Indeed, if it could be shown that distinctive rhythms performed by peoples apparently so isolated from one another over such a long time period have a common origin, this knowledge would be an extremely valuable resource for understanding both the evolution of African music and cultural evolution generally. Mr. Poole deserves considerable credit for grasping the significance of such patterns as possible markers of musical ancestry.

Before continuing, however, I feel it necessary to nitpick a bit over the term "timeline," which typically refers to rhythmic patterns performed solo, either on an iron bell, as already indicated, or some other percussion instrument chosen for that purpose, such as the wooden claves used typically in Latin America. Whether rhythmic patterns found among Pygmy and Bushmen groups in very different musical and social contexts, traditionally produced communally by handclaps or the beating of sticks or rattles, 
should also be called "timelines" would appear to be an open question, as this relationship has, to my knowledge, never been researched, much less established. Indeed, if it could be established that the clapping rhythms so commonly found among Pygmy and Bushmen groups were indeed foundational for the timelines so commonly found throughout sub-Saharan Africa, that would be a major piece of historical reconstruction. Personally, I do believe that very likely to be the case, and for that reason don't see Poole's usage as a serious drawback, especially since it is based on precedents established by authoritative figures such as Simha Arom. Nevertheless, until the deep history of the timeline has been sufficiently researched, the term as used in this context should be taken as more of an insight than an established fact.

Minor quibbles aside, this is exactly the sort of highly focused and objective comparative study I'd been hoping for in the wake of my own more general and subjectively formulated efforts in a similar direction. Indeed, this paper represents a significant contribution to the aforementioned debate over key issues of musical and cultural history.

\section{MATERIALS AND METHODS}

Poole's research is valuable first of all as a thorough review of the basic principles behind the rhythmic patterns under study and their application in various contexts. In the section titled "Musical Materials and Methods," he identifies his sources and outlines his method. Following in the wake of fundamental research by Simha Arom, Willi Anku, Gerhard Kubik, Godfried Toussaint, and others, he provides some valuable observations, both general and specific, regarding the nature of Pygmy and Bushmen "timelines," the various manners in which they can be represented, and how they can be compared. Especially significant is the fact that each can be notated in binary terms, as a string of 1's and 0's. While certain similarities between music and mathematics have often been noted, this is one of the few cases where the two literally converge. For Poole, representation in binary terms is especially useful as it enables comparisons according to "the well-known edit-distance or Levenshtein-distance algorithm," providing "a measure of the similarity between the binary representations of two rhythms," which in turn enables the construction of a distance-matrix leading, ultimately, to the generation of a phylogenetic tree.

\section{SIMILARITY AND DIFFERENCE}

The following section, titled "Structural Similarity and Difference," is largely devoted to a description of his Table 1, presenting 31 "timelines" drawn from his analyses of rhythmic patterns found among the Pygmy and Bushmen groups that are the object of his study. In addition to differentiating between western Pygmy, eastern Pygmy, and Bushmen timelines, he further "differentiates between timelines that divide the tactus ... into 3 and those that divide the tactus into 2 or 4, typically referred to as "ternary" and "binary" rhythms." I'll have more to say about this distinction presently.

\section{RHYTHMIC ODDITIES}

There follows a long and interesting, though somewhat confusing, segment devoted to what Simha Arom refers to as "rhythmic oddities," defined as timelines "segmented as closely as possible to the central dividing point' resulting in two parts 'composed of an odd number of minimal values,"” (Arom, 1989, p. 94). In the course of this discussion, Poole notes "that the rhythmic oddity model [seems] relevant to the timelines used by Pygmy groups from the Western region of Central Africa but is not applicable to the San Bushmen, who live in Namibia and Botswana some 2500km to the south, or Mbuti Pygmies who $1600 \mathrm{~km}$ to the west." Whether this distinction is significant or simply a coincidence remains unclear. It should be noted, by the way, that Arom's confusing terminology does not refer to the "oddity" of such timelines in the sense of their being "odd" or "strange," but to the "odd number of minimal values" contained therein.

\section{A GENERATIVE APPROACH}

The following segment is devoted to the application of "Generative Theory" to the comparative study of "triple-pulse" timelines. While Poole makes no direct reference to Noam Chomsky, this segment of his paper is deeply indebted to the theories of this pioneering linguist, whose ideas regarding "deep structure" and "generative linguistics" have been applied to the study of African rhythm by Kofi Agawu, Willi Anku, 
and others. For Poole, such research "suggests that many timelines used in sub-Saharan African musics may be generated from a few basic patterns." He then goes on to review fascinating research in this area by scholars such as Agawu, Pressing, Toussaint, Peñalosa, and Novotney, based on studies by Jones, Kubik, Fürniss, and others. While this segment of his paper is especially dense and sometimes difficult to follow, it offers an extraordinarily rich and detailed picture of these rhythmic patterns and their distribution that I, for one, found especially interesting and valuable.

Ultimately, Poole identifies a single pattern (x.x .x., or 222) as a "seed" for "all triple-pulse Pygmy and Bushmen timelines," serving as "not only an important point of connection between Pygmy and Bushmen cultural groups, but also [suggesting] that there are structural similarities between Pygmy/Bushmen triple-pulse timelines and those used in other parts of sub-Saharan Africa and related musics around the world." And here I wish Poole had elaborated a bit because, for me, the distinction between "ternary" and "binary" cries out for further clarification. According to Godfried Toussaint, "the word ternary (also triple meter) is used to describe those rhythms that have the property that the number of pulses contained in the time span . . . is divisible by three." (Toussaint, 2003, p. 26) Simple enough. But what bearing does such a distinction actually have on any given musical performance? Are ternary timelines actually the equivalent of "triple meter," as Toussaint's definition assumes? Are African rhythms actually based on an underlying meter, and if so, can such "meters" be compared to the meters found so typically in European music?

Considering the importance he attaches to this distinction, Poole's analysis strikes me as overly vague. A pattern designated as 222 tells us nothing, in itself, about any underlying subdivision of the pulse (assumed in this case to be ternary), yet one-dimensional representations of this kind are the basis for Poole's mathematical comparisons. What this issue boils down to is the question of whether timelines in general can be regarded as one- or two-dimensional, or, to put it another way, whether or not African rhythms are based, as are so many Western rhythms, on an implied, regularly recurring, underlying meter. This is a question I've often asked myself, because so much African music seems based on continual conflicts between two different underlying patterns, triple and duple, roughly analogous to an underlying metric, apparently. Yet, despite the tensions we hear so often between surface and background in African rhythms, I do not hear such rhythms as metric, because my perception of these patterns is continually shifting in a manner analogous to the way in which "figure-ground" relations become unstable in, say, a cubist painting. For me at least, the perception of a timeline can easily shift from a triple to a duple basis during the same performance, depending on a range of subjective factors.

While I am certainly not an expert on African rhythm, and the issue of timeline "meter" is not central to Poole's investigation, there are aspects of his analysis, such as his invocation of the 222 pattern as fundamentally "ternary," that could be less cryptic if this issue had been taken into consideration.

\section{MAXIMAL EVENNESS}

Poole attaches some importance to a concept labeled "maximal evenness," drawing especially on the work of Godfried Toussaint and Justin London. According to Poole, timelines are "maximally even" if "the strokes are distributed as evenly as possible within the cycle." As Poole explains, Toussaint's research in this area, as with so much of his work on African music, is characterized by the employment of mathematical principles to define evenness, asymmetricality, irregularity and even "surprise." London, on the other hand, is oriented more in the direction of percept psychology. His principal concerns are with the attention of the listener (and presumably also the dancer), with certain patterns more likely to permit $\mathrm{him} / \mathrm{h}$ er to "differentiate among events of varying salience" (London, 2004, p. 114). London also associates maximally even patterns with "efficient motor behaviours," implying that timelines with more evenness encourage "smooth, well formed" movements (London, 2004).

What most interests Poole is the question of how the principle relates to the construction of Pygmy and Bushmen timelines, and here he finds some interesting similarities and differences. Pygmy timelines tend to be "denser" than those of Bushmen, but both appear, nevertheless, "to be organized according to the principles of maximal evenness," which moreover appears to be a common feature of many timelines found throughout sub-Saharan Africa. 


\section{CULTURAL CONTEXT}

The apparent necessity of evaluating any musical style within its "cultural context" has been stressed over and over again in the literature of ethnomusicology. However, my own research has led me to believe that the importance of context, as far as performance style is concerned, has been considerably over-rated. Time and again we see very similar musical styles employed in widely varying contexts, both within a given social group and when comparing different groups. It is thus personally gratifying to learn that Poole's research on Pygmy and Bushmen timelines has led him to a similar conclusion.

After considering a range of different instances among various Pygmy and Bushmen groups, as recounted by Michelle Kisliuk, John Brearley, Colin Turnbull, and Richard Katz, Poole notes that, first, "individual timelines are multi-purpose and do not appear to be associated with one particular single song category, event or occasion, both within and across Pygmy and Bushmen cultures," and second, "far from being frozen or static, [Pygmy and Bushmen cultures] have and continue to adapt existing musical materials present within their own musical heritage and incorporate those from their Pygmy/Bushmen and non-Pygmy/non-Bushmen (mainly Bantu-speaking) neighbours to create new ones." In sum, after some very interesting descriptions of the various contexts in which Pygmy and Bushmen "timelines" can be found, it becomes evident that such patterns are "multi-purpose" and flexible, and cannot be easily associated with any particular "category, event or occasion."

\section{CONTINUITY AND CHANGE}

The following section, titled "Continuity, Change and Resilience," deals with an aspect of Pygmy/Bushmen culture that I have always found especially relevant, i.e., a stubborn resistance to change with respect to certain core values and behaviors. In this respect, the comparative study of Pygmy and Bushmen musical practices, including the preservation of basic "timeline" patterns in both traditions, is especially instructive, as Poole very clearly demonstrates. While, as he acknowledges, "[v]ariation on the basic stroke patterns through improvisation or adaptation in order to accommodate new musical influences is likely," I fully concur in his conclusion that, nevertheless, "deep-rooted radical change to their fundamental structure seems improbable."

\section{A PHYLOGENETIC TREE}

The final section of this very ambitious and thorough essay is especially intriguing. Inspired by the methods of population geneticists, who systematically compare genetic haplotypes in an effort to retrace human lineages, Poole presents a "phylogenetic tree" of rhythmic patterns, based on a similarity matrix made possible by the fact that all these patterns can be expressed using binary numbers, and can thus be compared in strictly mathematical terms. He describes this tree as "a visual representation of the hierarchical distance between all timelines and their cluster relationships."

As with an earlier attempt at producing a phylogeny of mainstream African rhythms along similar lines by Godfried Toussaint (2003), my response is mixed. On the one hand, I find the "tree" Poole has produced rather fascinating. I heartily applaud his brave effort to carry the mathematical implications of his method to the limit, and am in complete sympathy with his effort to use phylogenetic methods in the hope of identifying a primal rhythmic "seed" from which all the various patterns may have evolved. As he is, of course, aware, similar methods have been applied in many different areas of cultural studies, from the evolution of Polynesian outrigger canoes to the development of certain musical instruments, and it seems to me that such efforts hold great promise - so long as their limitations are fully acknowledged.

On the other hand, there is a significant difference between the phylogenetic trees produced by population geneticists and those produced by students of culture. For one thing, the mechanisms necessary to produce the changes tracked by geneticists are well understood. Nucleotides mutate spontaneously and at random, and once they mutate that mutation usually remains fixed in place for all future generations. Population geneticists can thus move systematically backward, from the most recent branches to the oldest ones, by following the trail of each such mutation to its source. In contrast, the mechanisms by which cultural elements can be said to "mutate," assuming they do at all, are not very well understood, and all too frequently there is no clear pathway from present to past. Thus we have no way of knowing whether the mathematically defined similarities produced by Poole's methodology represent real historical 
developments or are purely theoretical. Nevertheless, in spite of such limitations, I find both Poole's phylogenetic tree and the comparative research behind it of genuine interest. Especially intriguing is the cluster of exclusively BaAka "timelines" stemming from the upper-left-most branches of his Figures $4 \mathrm{~b}$ and $4 \mathrm{c}$.

We must, of course, recognize that the phylogenetic trees produced by population geneticists have nothing to do with "evolution" in the usual sense of the word. In fact the markers used for this purpose are generally neutral markers, thought to be free of any influences from evolutionary adaptation. What researchers in this field are tracking is not evolution, but lineage - i.e., ancestry. Poole seems perfectly aware that what he is looking for is "ancestry" in a very similar sense, but when we are dealing with cultural practices it is difficult to prevent notions of evolution from creeping in unannounced. If, for example, it might be possible to identify one particular cultural "seed" that predated all the others tens of thousands of years ago, one would then want to speculate regarding the means by which this seed "evolved" into all its many, presumably more complex, descendants over all those years. But unlike the production of a genetic lineage, there is no clear equivalent in culture for a mutation. Genetic mutations appear spontaneously and for no reason, and are, moreover, embodied physically within a DNA molecule, while cultural elements are generally thought to be meaningful responses to environmental influences, and, aside from archaeological artefacts, are often incorporeal. If, for example, 222 could be considered the prototype for all the many variants found among African timelines, it would not be at all easy to speculate regarding the mechanism by which it could have evolved (or "mutated") from 222 to, let's say, 22122.

It is indeed fascinating to posit some sort of primal "seed" for any element of culture, and speculate as to how that seed might have sprouted to produce all its many descendents over the years. As far as timelines are concerned, however, it seems to me that the "seed" Poole is looking for is much more likely to take the form of a basic principle rather than a specific rhythmic pattern. I do think he is on the right track, however, by positing 222, against an underlying triple-pulse, as a possible "seed." My own preference would be to regard the basic principle of two-against-three polyrhythm as the fundamental basis for African rhythms generally, rather than any single "timeline." As should be clear from his Table One, almost all the timelines juxtapose duple and triple elements, strongly suggesting that it is the conflict between the two that is basic.

Poole continues with a brief summary of developments in population genetics suggesting "an ancient common origin" among all Pygmy and Bushmen groups. In this context, I'm gratified to see references to some of my own speculations regarding possible analogies between musical and genetic ancestry. While clapping patterns were not considered in my own research (an oversight, I must admit), Poole notes that my approach "is an intriguing one that could explain the timeline data presented above," going on to speculate that "the 222 seed pattern" he's identified could represent "the music of a common ancestral group." I see no reason to dispute such a hypothesis, very much in line with my own thinking, but must concur when Poole reminds us that "extreme caution is required when reconstructing complex cultural practices such as music that may originate far back in the Paleolithic."

In the light of my own research into the relation of Pygmy and Bushmen musical practices (Grauer, 2003, 2006, 2009), Poole's conclusion that, "despite variations in cycle length and the number and placement of strokes, Pygmy and Bushmen timelines are highly interrelated" is not at all surprising and, in view of his unusually objective, precise and thoroughgoing methodology, especially convincing. I agree, moreover, that Poole's approach "highlights important relationships between some Pygmy/Bushmen timelines and those used in music in other parts of sub-Saharan Africa, the Caribbean and beyond that could be explored in future studies," and would enthusiastically urge him to do so.

\section{ACKNOWLEDGEMENTS}

This article was copyedited by Dana Lauren DeVlieger and layout edited by Kelly Jakubowski.

\section{NOTES}

[1] Dr. Grauer may be contacted via email at victorag@verizon.net 


\section{REFERENCES}

Arom, S. (1989). Time structure in the music of Central Africa: Periodicity, meter, rhythm and polyrhythmics. Leonardo, 22(1), 91-99. https://doi.org/10.2307/1575146

Grauer, V. (2006). Echoes of our forgotten ancestors. The World of Music, 48(2), 5-58.

Grauer, V. (2009). Concept, style, and structure in the music of the African Pygmies and Bushmen: A study in cross-cultural analysis. Ethnomusicology, 53(3), 396-424.

Grauer, V. (2011). Sounding the depths: Tradition and the voices of history. CreateSpace.

London, J. (2004). Hearing in time: Psychological aspects of musical meter. New York: Oxford University Press. https://doi.org/10.1093/acprof:oso/9780195160819.001.0001

Toussaint, G. T. (2003). Classification and phylogenetic analysis of African ternary rhythm timelines. BRIDGES: Mathematical Connections in Art, Music and Science, University of Granada, Spain. 